\title{
The emergence of dynamic phenotyping
}

\author{
Daniel Ruderman (D)
}

Received: 14 September 2017 / Accepted: 18 September 2017 / Published online: 23 September 2017

(C) Springer Science+Business Media B.V. 2017

Recent experiments have revealed the importance of discerning temporal response behaviors in single cells. Such "dynamic phenotypes" cannot be seen when averaging measurements across many cells because different behaviors blur together. Many such results focus on changes in protein abundance, intracellular localization, and cell shape in response to stimuli, processes that require tracking living cells for minutes to hours. These discoveries have been enabled by live cell microscopy and analysis techniques that have become available only recently. In a recent Editorial (Wang et al. 2017), Wang et al. described many methods for assessing single-cell phenotypes. Here, I focus on how the cross-disciplinary nature of these techniques will be central to their advancement.

The best characterized example of a dynamic phenotype is the abundance changes of p53 in response to DNA damage. Based on Western blot studies, it was known that strong DNA damage causes damped multihour p53 oscillations, whose amplitude increases with damage (Bar-or et al. 2000). By looking at the dynamics of individual cells instead of populations using live cell imaging, Lahav et al. (2004) found something unexpected: single cells themselves do not have damped oscillations. Instead, cells have undamped oscillations with

\section{Ruderman $(\bowtie)$}

The Lawrence J. Ellison Institute for Transformative Medicine, University of Southern California, Los Angeles, CA 90089-9075, USA

e-mail: Ruderman@usc.edu varying numbers of cycles. A Western blot simply averages these varied responses into a damped oscillation. The increased response amplitude with DNA damage is also a population effect: higher DNA damage pushes more cells to longer oscillations, which add up in phase to a larger population amplitude. Thus taking the population average behavior as true for single cells can be misleading.

Dynamic phenotypes also relate to disease and therapeutic response. In HCT-116 cells, those with fast nuclear p53 accumulation after cisplatin exposure undergo apoptosis, whereas those with slow accumulation survive (Paek et al. 2016). In single lung cancer cells, early intracellular spatio-temporal dynamics correlate with TNF- $\alpha$ sensitivity (Loo et al. 2017). In type 2 diabetes, pulsatile release of insulin by beta islet cells is impaired (O'Rahilly et al. 1988). Yang et al. (2017) recently showed that altering p53 dynamics changes cell fate, demonstrating a causal link to response phenotype. Thus it may be valuable to characterize such disease states by how dynamical processes deviate from normal, which we might call "dynopathy." Such aberrant dynamics and signaling networks are in fact disease targets in their own right: Stewart-Ornstein and Lahav (2017), recently employed phenotypic profiling to discover small molecules that modulate p53 dynamics.

Of the many technical advances that have led to these discoveries, which are most important? High content screening of live cells, the delivery of time varying stimulation, and advanced microscopy image analysis platforms for tracking cells have all been critical. See (Gaudet and Miller-Jensen 2016; Handly et al. 2016; 
Bougen-Zhukov et al. 2016; Spiller et al. 2010) for reviews. Because many of these techniques are immature, it is important to rigorously assess data quality, preferably in an automated fashion (Patsch et al. 2016). Computational models have also provided key insights. For example, Mönke et al. (2017) used an ordinary differential equation model to replicate the heterogeneous dynamics of p53 across cells. They computationally predicted and then experimentally verified that Wip1 level is a major determining factor of heterogeneous cellular phenotypes. Thus dynamic phenotype research not only calls for advanced technology, but also interaction between disciplines.

Despite these advances, one key aspect seems to be missing from dynamic phenotyping. Because dynamic phenotypes are often measured across hours to days, it may be important to account for circadian rhythms, which have not been well characterized within single cells. It is known that cells have intrinsic circadian clocks which modulate their transcriptomes and dynamically impact phenotype (Bass and Lazar 2016). Extrinsic disruption of circadian rhythm in people (e.g., altered meal times, photic stimulation) contributes to metabolic disease and is linked to cardiovascular disease and cancer (Roenneberg and Merrow 2016). Although circulating hormones, core body temperature, glucose, and immune function all undergo cyclic variation, most in vitro research is carried out in static culture conditions. These conditions lack both synchronized cell clocks and the dynamic microenvironment. How could this be remedied? Multiple factors can synchronize cell clocks, including temperature, glucocorticoids, and serum shock. Experiments could be carried out under varying temperature and dynamic media composition (e.g., using microfluidic platforms). Recent experimental advances have enabled the measurement of singlecell dynamics in complex microenvironments, such as co-culture (Garvey et al. 2016) and tissue explants (Lande-Diner et al. 2015) and can advance our understanding of circadian phenotypes under more realistic conditions.

Other outstanding areas for advancing dynamic phenotype research include:

- Determining whether computational models can predict the phenotypes of cells at a quantitative level. Many models predict the existence of different classes of phenomena, but cannot necessarily match a given cell's behavior. Applications such as Virtual
Cell (Resasco et al. 2011) merge intracellular spatiotemporal data with partial differential equations to quantitatively model dynamical behavior. Simulation results can be numerically compared with live cell imaging data to test hypotheses (Neves et al. 2009). Random influences can also be included to quantify the impact of noise and model parameter changes on dynamic phenotypes (Eldar and Elowitz 2010). These modeling approaches allow us to describe how well we understand dynamic phenotypes in a quantitative manner.

- Developing new theories to identify key response variables for investigating dynamic phenotypes. Current theories of dynamic signaling networks focus on notions of efficiency (Behar et al. 2007) and reliability (Tostevin et al. 2012). However, there is little consensus on how cell populations' phenotypic heterogeneity should be interpreted. Is the average phenotype, the variability in phenotype, or the extreme phenotype most important? Rare cells with extreme phenotypes are often resistant, survive therapy, and cause disease recurrence in both cancer (Shaffer et al. 2017) and infectious disease (Metcalfe et al. 2016). Might their presence be inferred from the tail shapes of dynamic phenotype distributions? Approaches that derive the entire phenotype distributions from data (Cheng et al. 2015) may ferret out such extreme and outlier behaviors.

- Building multidisciplinary dynamic phenotype research teams. While the research questions come from the biological sciences, much of the execution relies on other highly technical disciplines. These include quantitative live cell imaging, time series analysis, computational modeling, and statistical testing on multi-dimensional data sets. Finding contributors who bridge multiple such skills and comprehend the biological questions remains a key challenge for research groups. It is best met by ensuring that our educational systems can develop these talented researchers and instill within them a sense of meaning from translational research.

It is increasingly clear that the final state of a cell's stimulus response is not the whole story. How it gets there also matters. Tools with more accurate data collection, more spatio-temporal detail, and more physiological dynamic microenvironments are becoming rapidly available, making dynamic phenotyping an exciting multidisciplinary field. We must equip ourselves with 
the right biological systems, clever questions, and capable quantitative researchers to make efficient use of these new technologies. In turn, we should impel technology advancement to keep pace with our expanding needs as driven by the science.

\section{References}

Bar-or RL, Maya R, Segel LA, Alon U, Levine AJ, Oren M. Generation of oscillations by the $\mathrm{p} 53-\mathrm{Mdm} 2$ feedback loop: a theoretical and experimental study. 2000;2-7.

Bass J, Lazar MA. Circadian time signatures of fitness and disease. 2016;354(6315). https://doi.org/10.1126/science.aah4965.

Behar M, Hao N, Dohlman HG, Elston TC. Mathematical and computational analysis of adaptation via feedback inhibition in signal transduction pathways. Biophys J. 2007;93(3):80621. https://doi.org/10.1529/biophysj.107.107516.

Bougen-Zhukov N, Loh SY, Lee HK, Loo L-H. Large-scale image-based screening and profiling of cellular phenotypes. Cytom Part A. 2016:1-11. https://doi.org/10.1002/cyto. a.22909.

Cheng Z, Taylor B, Ourthiague DR, Hoffmann A. Distinct singlecell signaling characteristics are conferred by the MyD88 and TRIF pathways during TLR4 activation. Sci Signal. 2015;8(385):ra69-ra69. https://doi.org/10.1126/scisignal. aaa5208.

Eldar A, Elowitz MB. Functional roles for noise in genetic circuits. Nature. 2010;467(7312):167-73. https://doi.org/10.1038 /nature09326.

Garvey CM, Spiller E, Lindsay D, et al. A high-content imagebased method for quantitatively studying context-dependent cell population dynamics. Sci Rep. 2016;6(1):29752. https://doi.org/10.1038/srep29752.

Gaudet S, Miller-Jensen K. Redefining signaling pathways with an expanding single-cell toolbox. Trends Biotechnol. $2016 ; 34(6): 458-69$. https://doi.org/10.1016/j. tibtech.2016.02.009.

Handly LN, Yao J, Wollman R. Signal transduction at the singlecell level: approaches to study the dynamic nature of signaling networks. J Mol Biol. 2016;428(19):3669-82. https://doi. org/10.1016/j.jmb.2016.07.009.

Lahav G, Rosenfeld N, Sigal A, et al. Dynamics of the p53-Mdm2 feedback loop in individual cells. Nat Genet. 2004;36(2): 147-50. http://www.nature.com/doifinder/10.1038/ng1293

Lande-Diner L, Stewart-Ornstein J, Weitz CJ, Lahav G. Singlecell analysis of circadian dynamics in tissue explants. Mol Biol Cell. 2015;26(22):3940-5. https://doi.org/10.1091/mbc. E15-06-0403.

Loo L-H, Bougen-Zhukov NM, Tan W-LC. Early spatiotemporalspecific changes in intermediate signals are predictive of cytotoxic sensitivity to TNF $\alpha$ and co-treatments. Sci Rep. 2017;7(January):43541. https://doi.org/10.1038/srep43541.

Metcalfe JZ, Streicher E, Theron G, et al. Cryptic microheteroresistance explains M. tuberculosis phenotypic resistance. Am J Respir Crit Care Med. 2016;4(May):301-19.

Mönke G, Cristiano E, Finzel A, et al. Excitability in the p53 network mediates robust signaling with tunable activation thresholds in single cells. Sci Rep. 2017;7(November 2016):46571. https://doi.org/10.1038/srep46571.

Neves SR, Tsokas P, Sarkar A, et al. Cell shape and negative links in regulatory motifs together control spatial information flow in signaling networks. Cell. 2009;133(4):666-80. https://doi. org/10.1016/j.cell.2008.04.025.Cell.

O'Rahilly S, Turner RC, Matthews DR. Impaired pulsatile secretion of insulin in relatives of patients with non-insuilindependent diabetes. N Engl J Med. 1988;318(19):1225-30.

Paek AL, Liu JC, Loewer A, Forrester WC, Lahav G. Cell-to-cell variation in p53 dynamics leads to fractional killing. Cell. 2016:1-13. https://doi.org/10.1016/j.cell.2016.03.025.

Patsch K, Chiu C-L, Engeln M, et al. Single cell dynamic phenotyping. Sci Rep. 2016;6(October):34785. https://doi. org/10.1038/srep34785.

Resasco DC, Gao F, Morgan F, Novak IL, Schaff JC, Slepchenko BM. Virtual cell: computational tools for modeling in cell biology. Wiley Interdiscip Rev Syst Biol Med. 2011;4(2): 129-40. http://doi.wiley.com/10.1002/wsbm.165

Roenneberg T, Merrow M. The circadian clock and human health. Curr Biol. 2016;26(10):R432-43. https://doi.org/10.1016/j. cub.2016.04.011.

Shaffer SM, Dunagin MC, Torborg SR, et al. Rare cell variability and drug-induced reprogramming as a mode of cancer drug resistance. Nature. 2017;546(7658):431-5. https://doi. org/10.1038/nature22794.

Spiller DG, Wood CD, Rand DA, White MRH. Measurement of single-cell dynamics. Nature. 2010;465(7299):736-45. http://www.nature.com/doifinder/10.1038/nature09232

Stewart-Ornstein J, Lahav G. p53 dynamics in response to DNA damage vary across cell lines and are shaped by efficiency of DNA repair and activity of the kinase ATM. Sci Signal. 2017;10(476):1-11. https://doi.org/10.1126/scisignal. aah6671.

Tostevin F, de Ronde W, ten Wolde PR. Reliability of frequency and amplitude decoding in gene regulation. Phys Rev Lett. 2012;108(10):108104. http://eutils.ncbi.nlm.nih. gov/entrez/eutils/elink.fcgi?dbfrom=pubmed $\&$ id=22463459 \&retmode $=$ ref \&cmd $=$ prlinks

Wang W, Zhu B, Wang X. Dynamic phenotypes: illustrating a single-cell odyssey. Cell Biol Toxicol. 2017:1-5. https://doi. org/10.1007/s10565-017-9400-2.

Yang R, Huang B, Zhu Y, Li Y, Liu F, Shi J. Variable sensitivity to DNA damaging chemotherapeutic modulated by cell typedependent bimodal p53 dynamics. BioRxiv. 2017; https://doi.org/10.1101/149013. 